\title{
Phase 1 trial of avelumab (anti-PD-L1) in Japanese patients with advanced solid tumors, including dose expansion in patients with gastric or gastroesophageal junction cancer: the JAVELIN Solid Tumor JPN trial
}

\author{
Toshihiko Doi ${ }^{1}$ - Satoru Iwasa ${ }^{2} \cdot$ Kei Muro ${ }^{3}$. Taroh Satoh ${ }^{4} \cdot$ Shuichi Hironaka $^{5}$ - Taito Esaki ${ }^{6}$. Tomohiro Nishina ${ }^{7}$. \\ Hiroki Hara ${ }^{8} \cdot$ Nozomu Machida $^{9} \cdot$ Yoshito Komatsu $^{10} \cdot$ Yasuhiro Shimada $^{11} \cdot$ Satoshi Otsu $^{12} \cdot$ Shin Shimizu $^{13}$. \\ Morihiro Watanabe ${ }^{13}$
}

Received: 20 September 2018 / Accepted: 21 November 2018 / Published online: 4 December 2018

(c) The Author(s) 2018

\begin{abstract}
Background Avelumab is a human anti-PD-L1 IgG1 monoclonal antibody that has shown antitumor activity in several advanced cancers. We report results from JAVELIN Solid Tumor JPN, a phase 1 trial of avelumab in Japanese patients with advanced solid tumors with expansion in patients with advanced gastric cancer/gastroesophageal junction cancer.

Methods In the dose-escalation part, eligible patients had various previously treated metastatic or advanced solid tumors. In the dose-expansion part, patients had stage IV gastric cancer/gastroesophageal junction adenocarcinoma and disease progression after prior therapy that included a platinum and fluoropyrimidine agent. Patients received avelumab every 2 weeks intravenously at 3,10 , or $20 \mathrm{mg} / \mathrm{kg}$ during dose escalation and $10 \mathrm{mg} / \mathrm{kg}$ during dose expansion.

Results Among 17 patients who received avelumab in the dose-escalation part, no dose-limiting toxicities occurred, and the maximum tolerated dose was not reached. 40 patients were enrolled in the dose-expansion part, of whom $21(52.5 \%)$ had received $\geq 3$ prior lines of therapy for advanced disease. In these patients, the objective response rate was $10.0 \%(95 \%$ CI, 2.8-23.7\%) and median overall survival was 9.1 months (95\% CI, 7.2-11.2 months). Three of 40 patients (7.5\%) had a grade 3 treatment-related adverse event (alanine aminotransferase increase, anemia, and hyponatremia), and no grade $\geq 4$ treatment-related adverse events occurred. Five patients (12.5\%) had an immune-related adverse event (all grade 1/2).

Conclusions Avelumab showed acceptable safety in Japanese patients with advanced solid tumors and clinical activity in patients with advanced gastric cancer/gastroesophageal junction cancer and disease progression after chemotherapy.
\end{abstract}

Keywords PD-L1 · Gastric cancer · Phase $1 \cdot$ Japan · Avelumab

Electronic supplementary material The online version of this article (https://doi.org/10.1007/s10120-018-0903-1) contains supplementary material, which is available to authorized users.

Toshihiko Doi

tdoi@east.ncc.go.jp

1 National Cancer Center Hospital East, 6-5-1 Kashiwanoha, Kashiwa, Chiba 277-8577, Japan

2 National Cancer Center Hospital, Chuo-ku, Tokyo, Japan

3 Aichi Cancer Center Hospital, Chikusa-ku, Nagoya, Japan

4 Osaka University Hospital, Suita, Osaka, Japan

5 Chiba Cancer Center, Chuo-ku, Chiba, Japan

6 National Kyushu Cancer Center, Minami-ku, Fukuoka, Japan

\footnotetext{
Shikoku Cancer Center, Matsuyama, Ehime, Japan

Saitama Cancer Center, Kita, Adachi-gun, Saitama, Japan

Shizuoka Cancer Center, Sunto-gun, Shizuoka, Japan

10 Hokkaido University Hospital, Kita-ku, Sapporo, Japan

1 Kochi Health Sciences Center, Kochi, Japan

12 Oita University Hospital, Oita, Japan

13 Merck Serono Co., Ltd, Tokyo, Japan
} 


\section{Introduction}

Gastric cancer (GC) is the fifth most common cancer and the third leading cause of cancer-related death worldwide [1]. The incidence of GC is higher in Asian populations compared with the rest of the world, as shown by agestandardized incidence rates per 100,000 men and women, respectively, of 35.4 and 13.8 in East Asia [2] and 45.8 and 16.5 in Japan [1] compared with 17.4 and 7.5 worldwide [2]. Because of the high incidence rates of GC, screening is routine in Japan and several other Asian countries, and patients are diagnosed earlier than in non-Asian countries. In population studies of patients with $\mathrm{GC} /$ gastroesophageal junction cancer (GEJC), Asian patients appear to survive longer than non-Asian patients, which is likely due in part to earlier diagnosis but may also be due to biological factors [3-6]. GEJC is a cancer distinct from GC but with a similar biology and similar treatments in the advanced setting [7-9]. Recommended treatments for advanced GC/ GEJC are generally consistent among countries. First-line therapy usually consists of doublet or triplet chemotherapy including a platinum agent and fluoropyrimidine, with the addition of trastuzumab in patients with human epidermal growth factor receptor 2 (HER2)-positive tumors. Secondline options include taxane, irinotecan, or ramucirumab monotherapy, or paclitaxel plus ramucirumab. Currently, there is no globally accepted standard of care for third-line treatment [9-11]. Japanese guidelines for GC are comparable to those in other regions except for the recommendation of S-1 as fluoropyrimidine of choice for first-line treatment ( $\mathrm{S}-1$ remains an investigational agent in North America) and paclitaxel plus ramucirumab as a preferred option for second-line treatment $[11,12]$. Triplet regimens are not recommended for general practice in Japan [11]. The overall prognosis for patients with advanced GC/ GEJC remains poor, particularly in countries without early screening programs $[10,11,13]$, and treatment advances in recent years have been limited.

The immune checkpoint proteins programmed cell death 1 ligand 1 (PD-L1) and programmed cell death 1 protein (PD-1) appear to play significant roles in GC/GEJC pathobiology, with PD-L1 being expressed in $30-45 \%$ of GC/GEJC tumors $[14,15]$. In some studies, PD-L1 expression has been associated with a worse prognosis in GC/ GEJC, including an increased incidence of disease progression and shorter survival [16-18], although other studies have reported conflicting findings [14, 15]. In addition, PD-1 is upregulated on T cells in patients with GC [19], further suggesting that blockade of the PD-L1 and PD-1 interactions is a rational therapeutic strategy.

Antibodies targeting PD-L1 or PD-1, which can reactivate suppressed antitumor immune responses, have become an established part of treatment for various cancers, and these agents have also shown clinical activity in patients with GC/GEJC. In a recent phase 3 trial in 493 patients with advanced GC performed in Japan, South Korea, and Taiwan (ATTRACTION-2), nivolumab (anti-PD-1) administered as third- or later-line treatment was associated with longer overall survival (OS) than placebo (median, 5.26 vs 4.14 months; $P<0.0001$ ); objective response rates (ORRs) for nivolumab vs placebo were $11.2 \%$ vs $0 \%$, respectively [20]. Based on this study, nivolumab was approved in Japan, South Korea, and Taiwan for the treatment of patients with unresectable advanced or recurrent GC and disease progression after chemotherapy. In a cohort of the phase 2 KEYNOTE-059 trial, pembrolizumab was administered as third- or laterline treatment in patients with GC/GEJC $(n=259)$. The ORR was $11.6 \%$ overall, and was $15.5 \%$ and $6.4 \%$ in patients with PD-L1+ and PD-L1 - tumors, respectively [21]. Based on these results, pembrolizumab was approved by the US Food and Drug Administration for the treatment of patients with PD-L1+ GC/GEJC and disease progression after $\geq 2$ prior lines of therapy. However, the global phase 3 trial, KEYNOTE-061, did not demonstrate superior OS for second-line pembrolizumab compared with paclitaxel in patients with GC/GEJC (median, 9.1 vs 8.3 months; $P<0.0421$ ) [22].

Avelumab is a human IgG1 monoclonal antibody with a wild-type Fc region that blocks the PD-L1/PD-1 interaction [23]. In addition to reactivating adaptive immune responses by inhibiting this interaction, preclinical models show that avelumab can also induce innate effector cell functions, a characteristic not seen with other approved anti-PD-L1/PD-1 antibodies; thus, avelumab may engage both the adaptive and innate immune systems [24, 25]. Avelumab has been approved in various countries for the treatment of metastatic Merkel cell carcinoma and locally advanced or metastatic urothelial carcinoma with progression following platinumcontaining chemotherapy [26-28].

International studies of avelumab in patients with advanced GC/GEJC have been reported. In a cohort from a large phase 1 trial, avelumab showed antitumor activity and an acceptable safety profile when administered as secondline or switch-maintenance treatment to patients with or without disease progression after first-line chemotherapy [29]. In a recently reported phase 3 trial, JAVELIN Gastric 300, avelumab administered as third-line treatment for GC/GEJC did not show superior efficacy compared with physician's choice of chemotherapy (median OS, 4.6 vs 5.0 months; $P<0.81$ ) [30].

Here, we report data from a trial of avelumab in Japanese patients (JAVELIN Solid Tumor JPN), including dose escalation in patients with various advanced solid tumors and dose expansion in a cohort of patients with GC/GEJC. 


\section{Methods}

\section{Study design and patients}

JAVELIN Solid Tumor JPN is a phase 1, open-label, multicenter trial performed in Japan (NCT01943461). In the dose-escalation part, eligible patients had histologically or cytologically confirmed metastatic or locally advanced solid tumors for which no standard therapy existed or standard therapy had failed. Initial patients in the dose-expansion part had histologically or cytologically confirmed, unresectable, measurable, stage IV GC/ GEJC adenocarcinoma and disease progression after 1 or 2 prior chemotherapy regimens that included both platinum and fluoropyrimidine therapy. Following a protocol amendment, eligible patients had disease progression after both first- and second-line treatment that included platinum and fluoropyrimidine treatment followed by taxane or irinotecan treatment. In the dose-expansion part, patients with severe peritoneal metastases (defined as clinical ileus or subileus or moderate-severe ascites) were ineligible, and patients were required to have fresh or archival tumor samples (formalin-fixed, paraffin-embedded tissue) available. Other inclusion criteria in both parts of the trial included age $\geq 20$ years, Eastern Cooperative Oncology Group (ECOG) performance status of 0 or 1, estimated life expectancy $\geq 3$ months, and adequate hematologic, hepatic, and renal function. Exclusion criteria included central nervous system metastases, prior therapy with any antibody or drug targeting a T-cell coregulatory protein (immune checkpoint; eg, PD-L1/PD-1), or other anticancer therapy $\leq 30$ days before start of study treatment (14 days in the dose-expansion part). Any use of steroids was tapered before study treatment except in patients with adrenal insufficiency, who could continue treatment at a physiological replacement dose.

\section{Procedures and assessments}

The dose-escalation part was performed using a standard $3+3$ design with avelumab doses of 3,10 , and $20 \mathrm{mg} / \mathrm{kg}$ administered by 1 -h intravenous infusion every 2 weeks (Q2W). After analysis of tolerability and pharmacokinetics (PK), the dose-expansion part started enrollment and all patients received avelumab $10 \mathrm{mg} / \mathrm{kg} \mathrm{Q} 2 \mathrm{~W}$. All patients (dose-escalation and dose-expansion cohorts) continued to receive their assigned dose throughout the trial. Premedication with diphenhydramine $25-50 \mathrm{mg}$ and acetaminophen $650 \mathrm{mg}$ (modified based on local treatment standards and guidelines) was required 30-60 min before all infusions of avelumab. In the dose-escalation part, the first patient treated at each dose level was observed for 16 days (until $48 \mathrm{~h}$ after the second dose) for the occurrence of any dose-limiting toxicity (DLT) before the second patient began treatment. The second and third patients were treated $\geq 48 \mathrm{~h}$ apart. Adverse events (AEs) and laboratory abnormalities were classified and graded according to the National Cancer Institute Common Terminology Criteria for Adverse Events version 4.0. A DLT was defined as any grade $\geq 3 \mathrm{AE}$ that occurred during the first 3 weeks of treatment in the dose-escalation part (ie, the DLT observation period) and was considered related to avelumab treatment by the investigator or the sponsor. The maximum tolerated dose (MTD) was defined as the highest dose level at which $\leq 1$ of 6 evaluable patients experienced a DLT.

Patients were treated until progression or unacceptable toxicity or until other protocol-specified criteria for withdrawal were met. Treatment was discontinued for any grade $4 \mathrm{AE}$, except for single laboratory values out of the normal range that were deemed unrelated to study treatment, without clinical correlate, and that resolved in $\leq 7$ days with medical management. Treatment was also discontinued for any grade $3 \mathrm{AE}$ except for $(1)$ transient $(\leq 6 \mathrm{~h})$ influenza-like symptoms or pyrexia controlled with medical management; (2) fatigue, local infusion-related reaction (IRR), headache, nausea, or emesis that resolved to grade $\leq 1$ in $\leq 24 \mathrm{~h}$; (3) single laboratory values out of the normal range that were deemed unrelated to study treatment and without clinical correlate (excluding a grade $\geq 3$ increase in liver enzyme concentrations) that resolved to grade $\leq 1$ in $\leq 7$ days after medical management has been initiated; (4) tumor flare phenomena (local pain, irritation, or localized rash at a known or suspected tumor site); or (5) worsening of ECOG performance score to $\geq 3$ that did not resolve to $\leq 2$ by the last day of the following treatment cycle. Grade 2 AEs were managed by treatment delays; events that did not resolve to grade $\leq 1$ by the end of the following treatment cycle or that recurred led to permanent discontinuation of avelumab (except for hormone insufficiencies that could be managed by replacement therapy).

Safety assessments included documentation of AEs, physical examination, clinical laboratory tests (hematology, hepatic panels, and serum chemistry), and documentation of concurrent medications. A serious AE (SAE) was defined as any untoward event that was life-threatening, required hospitalization, resulted in disability, was a congenital anomaly, resulted in death, or was otherwise considered as medically important. Immune-related AEs (irAEs) were identified using a prespecified list of Medical Dictionary for Regulatory Activities (MedDRA) terms followed by a comprehensive medical review. IRRs were analyzed using an expanded definition that included both a prespecified list of MedDRA preferred terms (reactions occurring post-infusion on the 
same day or following day) and related signs and symptoms (based on specified MedDRA terms) that occurred on the day of infusion and resolved $\leq 2$ days.

Clinical activity was assessed by investigators using Response Evaluation Criteria in Solid Tumors (RECIST) version 1.1 and modified immune-related response criteria to determine the best overall response and progression-free survival (PFS) duration. Radiographic tumor assessments were performed at baseline and then every 6 weeks. For patients who had a partial response (PR) or complete response (CR), a confirmatory CT or MRI scan was done no sooner than 28 days after assessment and preferably at the scheduled 6-week interval. Blood samples for analysis of avelumab concentrations in serum were drawn $6 \mathrm{~h}$ before and at the end of infusion (peak value) during weeks 1, 3, 5, 7, and 13, and then every 6 weeks. In the dose-escalation part, additional samples were taken $0.5,1,2,4,6,12,24,36,48$, and $168 \mathrm{~h}$ after the first infusion.

In patients enrolled in the dose-expansion part, PD-L1 expression was assessed in fresh and archival tumor samples using a proprietary immunohistochemistry assay (Dako PD-L1 immunohistochemistry 73-10 pharmDx; Carpinteria, CA) based on an anti-PD-L1 rabbit monoclonal antibody clone (73-10) under license to Merck KGaA [31]. In this report, PD-L1 status was defined using a cutoff of $\geq 1 \%$ of tumor cells showing partial or complete membrane PD-L1 staining of any intensity.

\section{Outcomes}

In the dose-escalation part, the primary endpoint was occurrence of DLTs during the first 3 weeks of treatment. Secondary endpoints included safety and tolerability, best overall response per investigator assessment (defined as best response obtained among all tumor assessments after the start of treatment with avelumab until documented disease progression), immune-related best overall response (using modified immune-related response criteria derived from RECIST v1.1) [32, 33], PFS duration, OS, PD-L1 expression, immunogenicity, and PK profile.

\section{Statistical methods}

Planned enrollment in this study was $\leq 18$ patients in the dose-escalation part (per $3+3$ design) and $\leq 40$ patients in the dose-expansion part. For the dose-expansion part, the sample size was selected to provide a probability of $\geq 87 \%$ to observe any AEs occurring in $\geq 5 \%$ of patients, and to provide 95\% Clopper-Pearson CIs for potential ORRs (defined as the proportion of patients with a PR or CR), eg, $10 \%$ (95\% CI, 2.8-23.7\%) or 15\% (95\% CI, 5.7-29.8\%). Safety and activity were analyzed in all patients who received $\geq 1$ dose of avelumab. In the dose-escalation part, the DLT analysis set was defined as all patients whose data were used to implement the dose-escalation schedule; patients were required to have received all trial treatment administrations in the DLT observation period or to have stopped treatment because of a DLT in the DLT observation period. Change in the sum of target lesion diameters from baseline over time was evaluated in patients with a baseline tumor assessment and $\geq 1$ postbaseline assessment. Time-to-event endpoints were estimated with the Kaplan-Meier method, and CIs for the median were calculated using the Brookmeyer-Crowley method. $P$ values for the association between categorical variables were determined using Fisher's exact test. PK parameters were estimated using WinNonlin ${ }^{\circledR}$ (Certera; Princeton, NJ, USA) version 5.0 or higher.

\section{Results}

\section{Patients}

In the dose-escalation and dose-expansion cohorts, the data cutoff date was August 10, 2016. In the dose-escalation part, 17 patients with various advanced solid tumors received avelumab Q2W at doses of $3 \mathrm{mg} / \mathrm{kg}(n=5), 10 \mathrm{mg} / \mathrm{kg}(n=6)$, or $20 \mathrm{mg} / \mathrm{kg}(n=6)$ (Table 1). Two of the first three patients assigned to the $3 \mathrm{mg} / \mathrm{kg}$ cohort were subsequently found to have received steroids and were, therefore, not evaluable for DLT assessment; thus, two additional patients were treated at this dose level to achieve a cohort of three DLT-evaluable patients. Six patients were treated at 10 and $20 \mathrm{mg} / \mathrm{kg}$ to further investigate the safety of avelumab. All 17 patients had received prior therapy; $13(76.5 \%)$ and $6(35.3 \%)$ had received $\geq 3$ or $\geq 4$ prior lines for advanced disease, respectively. Median duration of avelumab treatment for the 3,10, and $20 \mathrm{mg} / \mathrm{kg}$ dose levels was $3.9,12.2$, and 2.8 months, respectively. Three patients ( 1 from each dose level) were still receiving treatment at data cutoff. The most common reason for treatment discontinuation was disease progression [ $3 \mathrm{mg} / \mathrm{kg}, n=3$ (60.0\%); $10 \mathrm{mg}, n=5$ (83.3\%); and $20 \mathrm{mg} /$ $\mathrm{kg}, n=4(66.7 \%)]$; other reasons were death $[3 \mathrm{mg} / \mathrm{kg}, n=1$ $(20.0 \%)]$ and patient decision to receive a different treatment [20 $\mathrm{mg} / \mathrm{kg}, n=1(16.7 \%)]$.

After analysis of safety and PK data, patients were enrolled in the dose-expansion part. Overall, 40 patients with advanced GC/GEJC, who had a median age of 63 years (range, 37-77 years), were treated with avelumab $10 \mathrm{mg} /$ kg Q2W (Table 1). Most patients $[n=35$ (87.5\%)] had $\mathrm{GC}$ and the remainder $[n=5(12.5 \%)]$ had GEJC. HER2 status was positive in 11 patients $(27.5 \%)$, negative in 20 patients (50.0\%), and equivocal or not available in 9 patients (22.5\%). All 40 patients had received prior therapy, including $\geq 3$ or $\geq 4$ prior lines for advanced disease in 21 patients $(52.5 \%)$ and 7 patients (17.5\%), respectively. Median 
Table 1 Baseline characteristics

\begin{tabular}{|c|c|c|c|c|}
\hline \multirow[t]{2}{*}{ Characteristics } & \multicolumn{3}{|c|}{ Dose escalation $(n=17)$} & \multirow[t]{2}{*}{ Dose expansion $(N=40)$} \\
\hline & $3 \mathrm{mg} / \mathrm{kg}(n=5)$ & $10 \mathrm{mg} / \mathrm{kg}(n=6)$ & $20 \mathrm{mg} / \mathrm{kg}(n=6)$ & \\
\hline Median age (range), years & $46(32-69)$ & $62(30-67)$ & $67(56-74)$ & $63(37-77)$ \\
\hline$<65$ years, $n(\%)$ & $3(60.0)$ & $5(83.3)$ & $2(33.3)$ & $22(55.0)$ \\
\hline$\geq 65$ years, $n(\%)$ & $2(40.0)$ & $1(16.7)$ & $4(66.7)$ & $18(45.0)$ \\
\hline \multicolumn{5}{|l|}{ Sex, $n(\%)$} \\
\hline Male & $3(60.0)$ & $4(66.7)$ & $3(50.0)$ & $29(72.5)$ \\
\hline Female & $2(40.0)$ & $2(33.3)$ & $3(50.0)$ & $11(27.5)$ \\
\hline \multicolumn{5}{|l|}{ ECOG performance status, $n(\%)$} \\
\hline 0 & $5(100.0)$ & $4(66.7)$ & $5(83.3)$ & $23(57.5)$ \\
\hline 1 & 0 & $2(33.3)$ & $1(16.7)$ & $17(42.5)$ \\
\hline Median time since first diagnosis (range), years & $2.9(1.9-6.1)$ & $4.5(1.3-7.7)$ & $2.9(1.3-22.3)$ & $1.5(0.5-9.1)$ \\
\hline $\begin{array}{l}\text { Median time since diagnosis of metastatic disease } \\
\text { (range), years }\end{array}$ & $1.5(0.3-6.1)$ & $3.1(0.8-7.7)$ & $2.9(1.3-14.6)$ & $1.4(0.1-8.4)$ \\
\hline \multicolumn{5}{|l|}{ Site of primary tumor, $n(\%)$} \\
\hline Breast & $1(20.0)$ & 0 & 0 & 0 \\
\hline Choroid & 0 & 0 & $1(16.7)$ & 0 \\
\hline Colon & $1(20.0)$ & 0 & 0 & 0 \\
\hline Esophagus & 0 & $1(16.7)$ & 0 & 0 \\
\hline Gastroesophageal junction & 0 & 0 & 0 & $5(12.5)$ \\
\hline Lung & 0 & $2(33.3)$ & $1(16.7)$ & 0 \\
\hline Nasal cavity & $1(20.0)$ & 0 & 0 & 0 \\
\hline Rectum & 0 & $1(16.7)$ & 0 & 0 \\
\hline Skin & $2(40.0)$ & 0 & 0 & 0 \\
\hline Small intestine & 0 & 0 & $1(16.7)$ & 0 \\
\hline Stomach & 0 & $2(33.3)$ & $3(50.0)$ & $35(87.5)$ \\
\hline \multicolumn{5}{|c|}{ Number of prior anticancer therapy lines for metastatic or locally advanced disease, $n(\%)$} \\
\hline 1 & $1(20.0)$ & 0 & 0 & $3(7.5)$ \\
\hline 2 & 0 & $1(16.7)$ & $1(16.7)$ & $14(35.0)$ \\
\hline 3 & $2(40.0)$ & $3(50.0)$ & $2(33.3)$ & $14(35.0)$ \\
\hline$\geq 4$ & $1(20.0)$ & $2(33.3)$ & $3(50.0)$ & $7(17.5)$ \\
\hline Missing & $1(20.0)$ & 0 & 0 & $2(5.0)$ \\
\hline PD-L1 expression ( $\geq 1 \%$ of tumor cells), $n(\%)$ & Not assessed & Not assessed & Not assessed & \\
\hline Negative & & & & $27(67.5)$ \\
\hline Positive & & & & $11(27.5)$ \\
\hline Not evaluable & & & & $2(5.0)$ \\
\hline
\end{tabular}

ECOG Eastern Cooperative Oncology Group

duration of avelumab treatment was 2.7 months (range, 0.5-21.4 months) and median follow-up was 19.3 months (range, 0.4-22.9 months). Two patients (5.0\%) were still receiving treatment at data cutoff. The most common reason for treatment discontinuation was disease progression $[n=33(82.5 \%)]$; other reasons were $\mathrm{AE}[n=4(10.0 \%)]$ and death $[n=1(2.5 \%)]$.

\section{Safety: dose-escalation cohort}

Of 15 patients in the dose-escalation cohort enrolled in the DLT analysis set ( $3 \mathrm{mg} / \mathrm{kg}, n=3 ; 10 \mathrm{mg} / \mathrm{kg}, n=6 ; 20 \mathrm{mg} /$ $\mathrm{kg}, n=6$ ), no patient had a DLT and the MTD was not reached. Of 17 patients in the full dose-escalation cohort, 16 patients (94.1\%) had an AE of any grade, of whom 11 patients $(64.7 \%)$ had a treatment-related AE (TRAE) of any grade: 3 patients $(60.0 \%)$ at $3 \mathrm{mg} / \mathrm{kg}, 5$ patients $(83.3 \%)$ at $10 \mathrm{mg} / \mathrm{kg}$, and 3 patients $(50.0 \%$ ) at $20 \mathrm{mg} / \mathrm{kg}$ (Table 2). No patient in the dose-escalation part had a grade $\geq 3$ TRAE.

Across all dose-escalation levels, IRRs identified via an expanded definition occurred in 5 patients $(29.4 \% ; 3 \mathrm{mg} /$ $\mathrm{kg}, n=1 ; 10 \mathrm{mg} / \mathrm{kg}, n=2 ; 20 \mathrm{mg} / \mathrm{kg}, n=2)$, all of which were grade 1 or 2 and occurred in patients in the DLT analysis set. Other TRAEs occurring in $\geq 20 \%$ of patients were 
Table 2 Treatment-related adverse events (TRAEs) occurring at any grade in $\geq 10 \%$ of patients at any dose level and infusion-related reactions in the dose-escalation cohort $(N=17)$

\begin{tabular}{llllc}
\hline & $3 \mathrm{mg} / \mathrm{kg}(n=5)$ & $10 \mathrm{mg} / \mathrm{kg}(n=6)$ & $20 \mathrm{mg} / \mathrm{kg}(n=6)$ & Overall $(N=17)$ \\
\hline Any TRAE, $n(\%)^{\mathrm{a}}$ & $3(60.0)$ & $5(83.3)$ & $3(50.0)$ & $11(64.7)$ \\
Rash maculopapular & $2(40.0)$ & $1(16.7)$ & $1(16.7)$ & $4(23.5)$ \\
Stomatitis & $1(20.0)$ & $3(50.0)$ & 0 & $4(23.5)$ \\
WBC count decreased & $1(20.0)$ & $2(33.3)$ & 0 & $3(17.6)$ \\
Anemia & $1(20.0)$ & 0 & $1(16.7)$ & $2(11.8)$ \\
Dermatitis acneiform & $1(20.0)$ & $1(16.7)$ & 0 & $2(11.8)$ \\
Headache & 0 & $1(16.7)$ & $1(16.7)$ & $2(11.8)$ \\
Pyrexia & 0 & $1(16.7)$ & $1(16.7)$ & $2(11.8)$ \\
Infusion-related reaction, $n(\%)^{\mathrm{b}}$ & $1(20.0)$ & $2(33.3)$ & $2(33.3)$ & $5(29.4)$ \\
\hline
\end{tabular}

No grade $\geq 3$ TRAEs occurred

$W B C$ white blood cell

${ }^{a}$ The incidence of treatment-related infusion-related reaction based on the single MedDRA preferred term is not listed.

${ }^{\mathrm{b}}$ Composite term; includes AEs categorized as infusion-related reaction, drug hypersensitivity, or hypersensitivity reaction that occurred on the day of infusion or day after infusion, in addition to signs and symptoms of infusion-related reaction that occurred on the same day of infusion and resolved within 2 days (including AEs classified by investigators as related or unrelated to treatment) rash maculopapular $[n=4(23.5 \%)]$ and stomatitis $[n=4$ $(23.5 \%)$ ]. Two patients $(11.8 \%)$ had an irAE: 1 patient $(3 \mathrm{mg} / \mathrm{kg}$ ) had grade 2 rash maculopapular, and 1 patient $(20 \mathrm{mg} / \mathrm{kg})$ had grade 3 aspartate aminotransferase increase and grade 1 alanine aminotransferase (ALT) increase. Two patients (11.8\%) had serious AEs, which were not treatmentrelated, and no patient had an $\mathrm{AE}$ that led to death.

\section{Safety: dose-expansion cohort}

All 40 patients in the dose-expansion cohort had an AE of any grade, of which 32 patients $(80.0 \%)$ had a TRAE of any grade (Table 3 ). IRRs identified via an expanded definition occurred in 12 patients (30.0\%); all were grade 1 or 2 , occurred at the first $(n=11)$ or second $(n=1)$ infusion, and did not lead to treatment discontinuation. Other TRAEs occurring at any grade in $\geq 10 \%$ of patients were pruritus $[n=6(15.0 \%)]$, pyrexia $[n=5(12.5 \%)]$, and rash $[n=4$ $(10.0 \%)]$. Three patients $(7.5 \%)$ had a grade 3 TRAE (ALT increase, anemia, and hyponatremia); no grade $\geq 4$ TRAEs occurred. Five patients (12.5\%) had an irAE, all of which were grade 1 or 2 (Table 3$)$. Pruritus $(n=3)$ and maculopapular rash $(n=2)$ were the only irAEs that occurred in $>1$ patient. Ten patients $(25.0 \%)$ had a serious AE, and three patients $(7.5 \%)$ had an AE that led to death [myocardial infarction, multiple organ failure, and aggravation of underlying disease (GC)]; none of these were treatment-related.

\section{Pharmacokinetics analyses}

All 57 patients were evaluable for PK analysis. In the doseescalation cohort (Online Resource 1), avelumab exposure in terms of maximum serum concentration observed
Table 3 Treatment-related adverse events (TRAEs; any grade in $\geq 10 \%$ of patients or grade $\geq 3$ in any patient), infusion-related reactions, and immune-related adverse events (AEs; any grade in any patient) in patients with GC/GEJC in the dose-expansion cohort $(N=40)$

\begin{tabular}{lrl}
\hline$N=40$ & Any grade & Grade 3 \\
\hline Any TRAE, n $(\%)^{\mathrm{a}}$ & $32(80.0)$ & $3(7.5)$ \\
Pruritus & $6(15.0)$ & 0 \\
Pyrexia & $5(12.5)$ & 0 \\
Rash & $4(10.0)$ & 0 \\
Anemia & $2(5.0)$ & $1(2.5)$ \\
Alanine aminotransferase increased & $1(2.5)$ & $1(2.5)$ \\
Hyponatraemia & $1(2.5)$ & $1(2.5)$ \\
Infusion-related reaction ${ }^{\mathrm{b}}$ & $12(30.0)$ & 0 \\
Any immune-related AE, $n(\%)$ & $5(12.5)$ & 0 \\
Pruritus & $3(7.5)$ & 0 \\
Rash maculopapular & $2(5.0)$ & 0 \\
Rash & $1(2.5)$ & 0 \\
Hyperthyroidism & $1(2.5)$ & 0 \\
Hypothyroidism & $1(2.5)$ & 0 \\
Secondary adrenocortical insufficiency & $1(2.5)$ & 0 \\
\hline
\end{tabular}

No grade $\geq 4$ TRAEs occurred

${ }^{a}$ The incidence of treatment-related infusion-related reaction based on the single MedDRA preferred term is not listed

${ }^{\mathrm{b}}$ Composite term; includes AEs categorized as infusion-related reaction, drug hypersensitivity, or hypersensitivity reaction that occurred on the day of infusion or day after infusion, in addition to signs and symptoms of infusion-related reaction that occurred on the same day as the infusion and resolved within 2 days (including AEs classified by investigators as related or unrelated to treatment) 
postdose $\left(C_{\max }\right)$ and area under the concentration-time curve from time $0-2$ weeks $\left(\mathrm{AUC}_{0-336 \mathrm{~h}}\right)$ after first administration increased in an approximately dose-proportional fashion between 3 and $20 \mathrm{mg} / \mathrm{kg}$ doses, and the estimated geometric mean apparent half-life $\left(t_{1 / 2}\right)$ ranged from 94 to $122 \mathrm{~h}$ (corresponding to 3.9-5.1 days). Mild drug accumulation following multiple infusions was observed (shown by an increase in mean $C_{\text {trough }}$ over time; Online Resource 2), consistent with the estimated $t_{1 / 2}$ from first administration and the Q2W dosing regimen. Median trough concentration levels $\left(C_{\text {trough }}\right)$ of avelumab showed a high degree of interpatient variability (coefficient of variation shown in Online Resource 1). Following administration of avelumab at $10.0 \mathrm{mg} / \mathrm{kg}$, median $C_{\text {trough }}$ appeared to be higher in the dose-escalation part (range, 20.6-54.7 $\mu \mathrm{g} / \mathrm{mL}$ ) than in the dose-expansion part (range, 14.12-22.13 $\mu \mathrm{g} / \mathrm{mL}$ ).

\section{Antitumor activity}

In the dose-escalation cohort, 3 patients (17.6\%) had a confirmed objective response (all PRs), comprising single patients with melanoma $(3 \mathrm{mg} / \mathrm{kg})$, esophageal squamous cell carcinoma (10 mg/kg), and GC (20 mg/kg). 11 additional patients had stable disease (SD) as best overall response.

In the dose-expansion cohort, the ORR was $10.0 \%$ (95\% CI, 2.8-23.7\%), including CR in 1 patient (2.5\%) and PR in 3 patients $(7.5 \%)$ (Table 4$)$. Of the 4 responding patients, 3 had received 2 prior lines of systemic therapy for advanced disease, and 1 had received 4 prior lines. The ORR in patients with 1,2 , or $\geq 3$ prior lines was $0 \%$ ( 0 of $3 ; 95 \%$ CI,

Table 4 Confirmed objective responses in patients with GC/GEJC in the dose-expansion cohort $(N=40)$

\begin{tabular}{ll}
\hline Response & $N=40$ \\
\hline Best overall response, $n(\%)$ & \\
Complete response & $1(2.5)$ \\
Partial response & $3(7.5)$ \\
Stable disease & $17(42.5)$ \\
Progressive disease & $17(42.5)$ \\
Not evaluable & $2(5.0)$ \\
ORR, \% (95\% CI) & $10.0(2.8-23.7)$ \\
Disease control rate, $\%$ & 52.5 \\
Immune-related best overall response, $n(\%)$ & \\
Complete response & $1(2.5)$ \\
Partial response & $3(7.5)$ \\
Stable disease & $21(52.5)$ \\
Progressive disease & $9(22.5)$ \\
Not evaluable & $6(15.0)$ \\
Immune-related ORR, \% (95\% CI) & $10.0(2.8-23.7)$ \\
\hline
\end{tabular}

$O R R$ objective response rate
$0.0-70.8 \%$ ), $21.4 \%$ (3 of $14 ; 95 \% \mathrm{CI}, 4.7-50.8 \%$ ), and $4.8 \%$ ( 1 of $21 ; 95 \%$ CI, $0.1-23.8 \%$ ), respectively. Of responding patients, 1 had a HER2+ tumor and 3 had HER2- tumors. Duration of response in the expansion cohort ranged from 2.8 to 17.7 months, with 1 response ongoing at data cutoff (Fig. 1a). An additional 17 patients (42.5\%) had a confirmed best overall response of SD, and the disease control rate was $52.5 \%$ (Table 4). The immune-related ORR was $10.0 \%$ (95\% CI, 2.8-23.7\%), and 21 (52.5\%) additional patients had a best overall response of immune-related SD. $\mathrm{A} \geq 30 \%$ tumor reduction occurred in $5(13.2 \%)$ of 38 evaluable patients (Fig. 1b, c). ORRs in patients with PD-L1+or PD-L1 - tumors were $27.3 \%$ (3 of 11 ; 95\% CI, 6.0-61.0\%) vs $3.7 \%$ ( 1 of 27 ; $95 \% \mathrm{CI}, 0.1-19.0 \%$; $P=.065$ ), respectively.

In the dose-expansion cohort, median PFS was 2.4 months (95\% CI, 1.4-2.8 months), and the 3-month PFS rate was $35.0 \%$ (95\% CI, 20.8-49.6\%) (Fig. 2a). Median PFS in patients with PD-L1+or PD-L1 - tumors was 1.4 months (95\% CI, 0.7-4.0 months) and 2.6 months (95\% CI, 1.4-2.8 months), respectively (Online Resource 3a). Median OS in all patients $(n=40)$ was 9.1 months $(95 \%$ CI, 7.2-11.2 months), and the 12-month OS rate was 31.0\% (95\% CI, 15.6-47.8\%) (Fig. 2b). Median OS in patients with PD-L1+ or PD-L1 - tumors was 10.9 months (95\% CI, 1.0 months-not estimable) and 9.1 months (95\% CI, 4.9-11.0 months), respectively (Online Resource 3b).

\section{Discussion}

In this single-arm study in Japanese patients, avelumab had an acceptable safety profile at doses up to $20 \mathrm{mg} / \mathrm{kg}$ Q2W. No DLTs were reported, and an MTD was not reached. The rate of grade 3 TRAEs was low ( $0 \%$ in the dose-escalation part and $7.5 \%$ in the dose-expansion part), and no grade $\geq 4$ TRAEs were reported.

PK parameters, including $C_{\text {trough }}, C_{\max }$, and end-of-infusion levels, were lower than seen in previous studies with the same dose levels in non-Asian patients, likely due to the lower mean body weight and associated higher relative blood volume in Japanese patients [34]. However, the safety profile of avelumab in Japanese patients in this study was consistent with that of previously reported studies in global populations $[23,32]$. The relatively lower $\mathrm{C}_{\text {trough }}$ in the doseexpansion cohort, compared with that in the dose-escalation cohort at the same dose level, may have been due to different disease characteristics in patients with GC/GEJC vs other tumor types, or it may have been a sampling artifact due to the small number of patients $(n=6)$ who received the $10 \mathrm{mg} /$ $\mathrm{kg}$ dose in the dose-escalation part.

Based on safety and PK findings from this study, and with consideration of PK and target occupancy data from an international phase 1 study of avelumab [23], the 
a

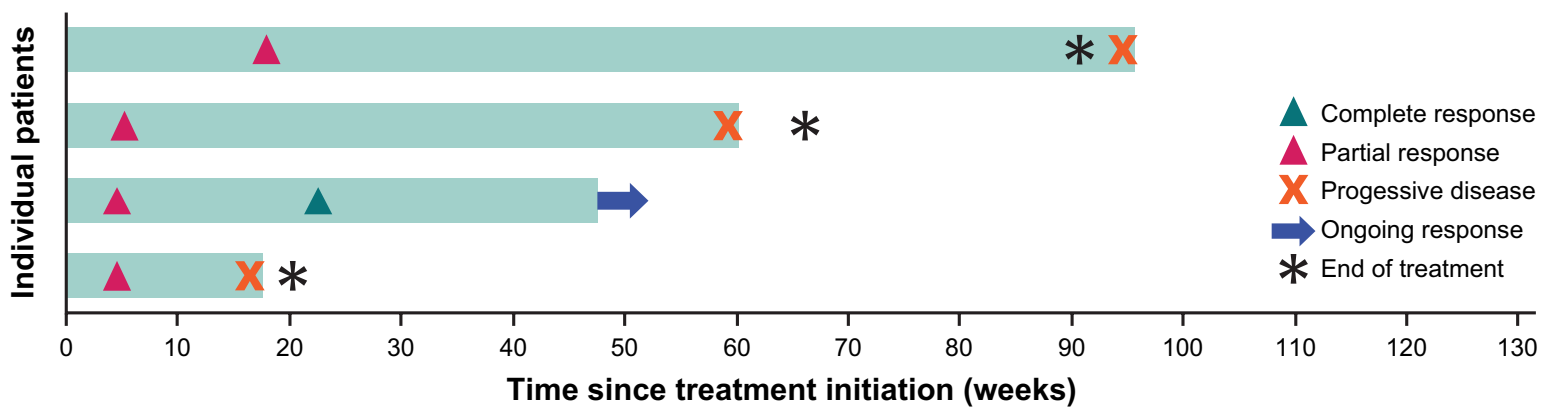

b

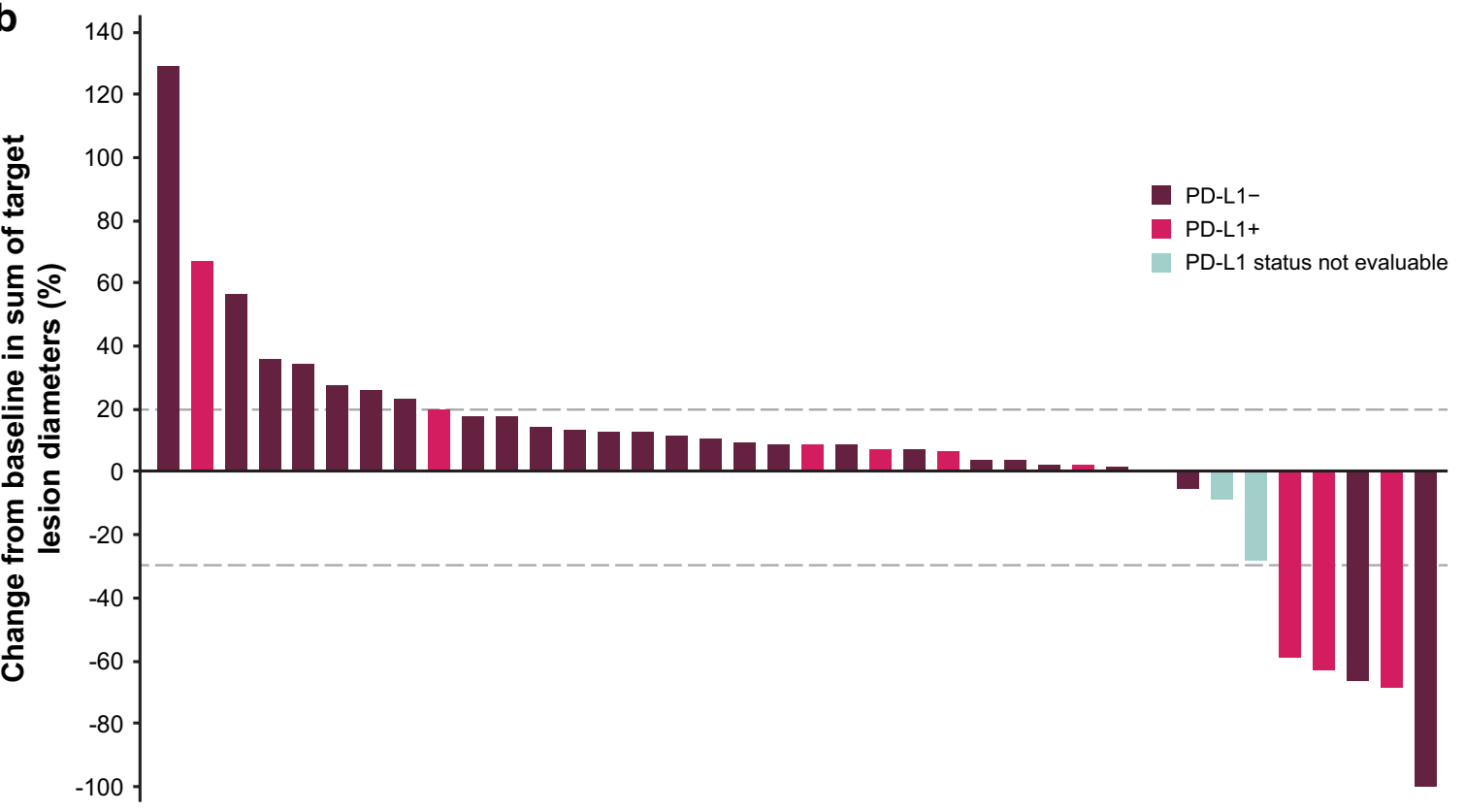

C

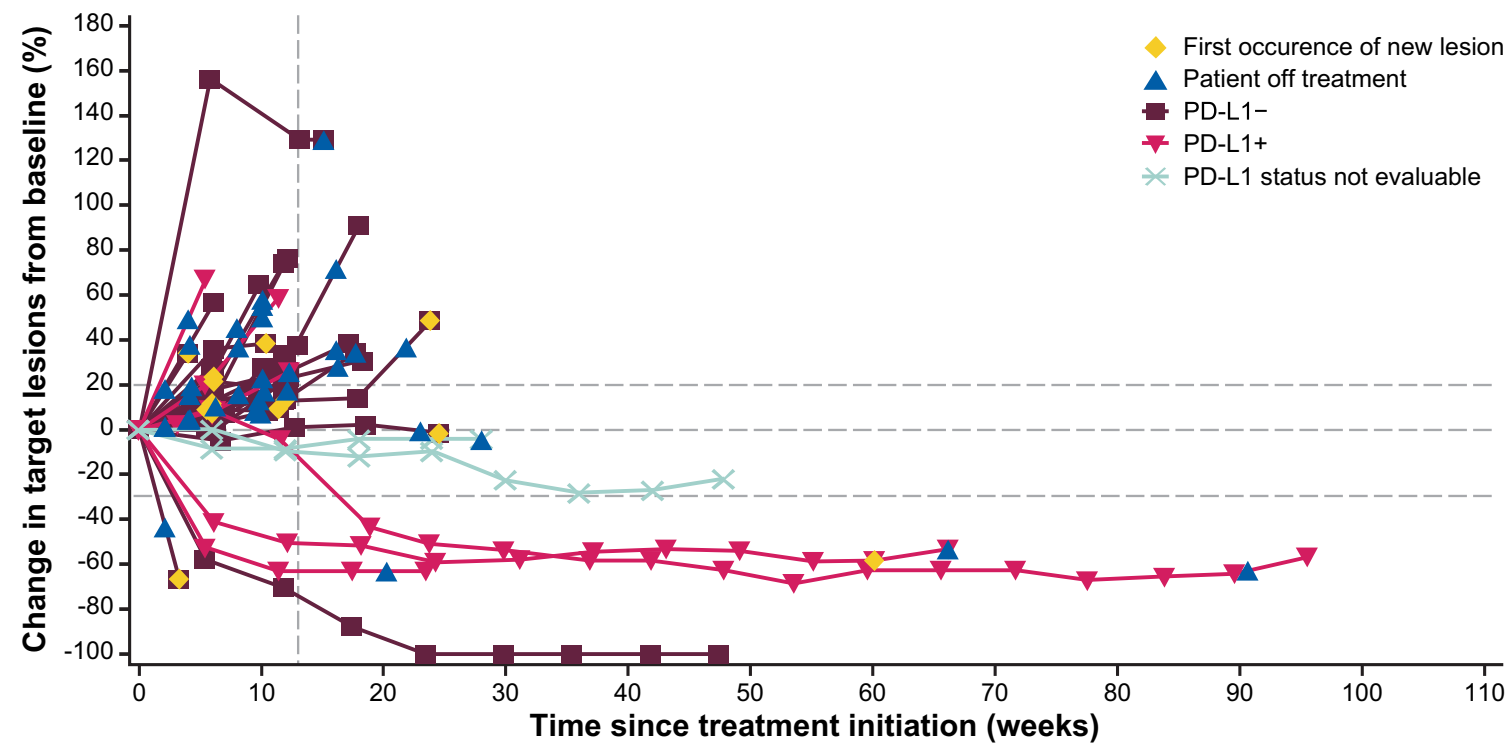


४Fig. 1 Antitumor activity of avelumab in patients with GC/GEJC in the dose-expansion cohort $(N=40)$. a Time to and duration of response in responding patients $(n=4)$. b Best change in target lesions from baseline by PD-L1 status ( $\geq 1 \%$ cutoff; $n=38$ evaluable). c Change in target lesions from baseline over time by PD-L1 status ( $\geq 1 \%$ cutoff; $n=38$ evaluable)

$10 \mathrm{mg} / \mathrm{kg}$ Q2W dose was selected for further study in a cohort of Japanese patients with advanced GC/GEJC. In these heavily pretreated patients, avelumab showed durable antitumor activity, including an ORR of $10.0 \%$ (including responses in patients with HER2+ and HER2- tumors), median PFS of 2.4 months, and median OS of 9.1 months (12-month OS rate of $31 \%$ ). The results of this trial are consistent with findings from a global cohort of patients with GC/GEJC treated in the JAVELIN Solid Tumor trial [29], and previous phase 1 and 2 studies of anti-PD-1 antibodies [21, 35, 36]. In our study, ORR was numerically higher in patients with PD-L1+ tumors, although responses were also seen in patients with PD-L1- tumors. It should be noted that PD-L1 expression status in this study was assessed using an assay (73-10) that is distinct
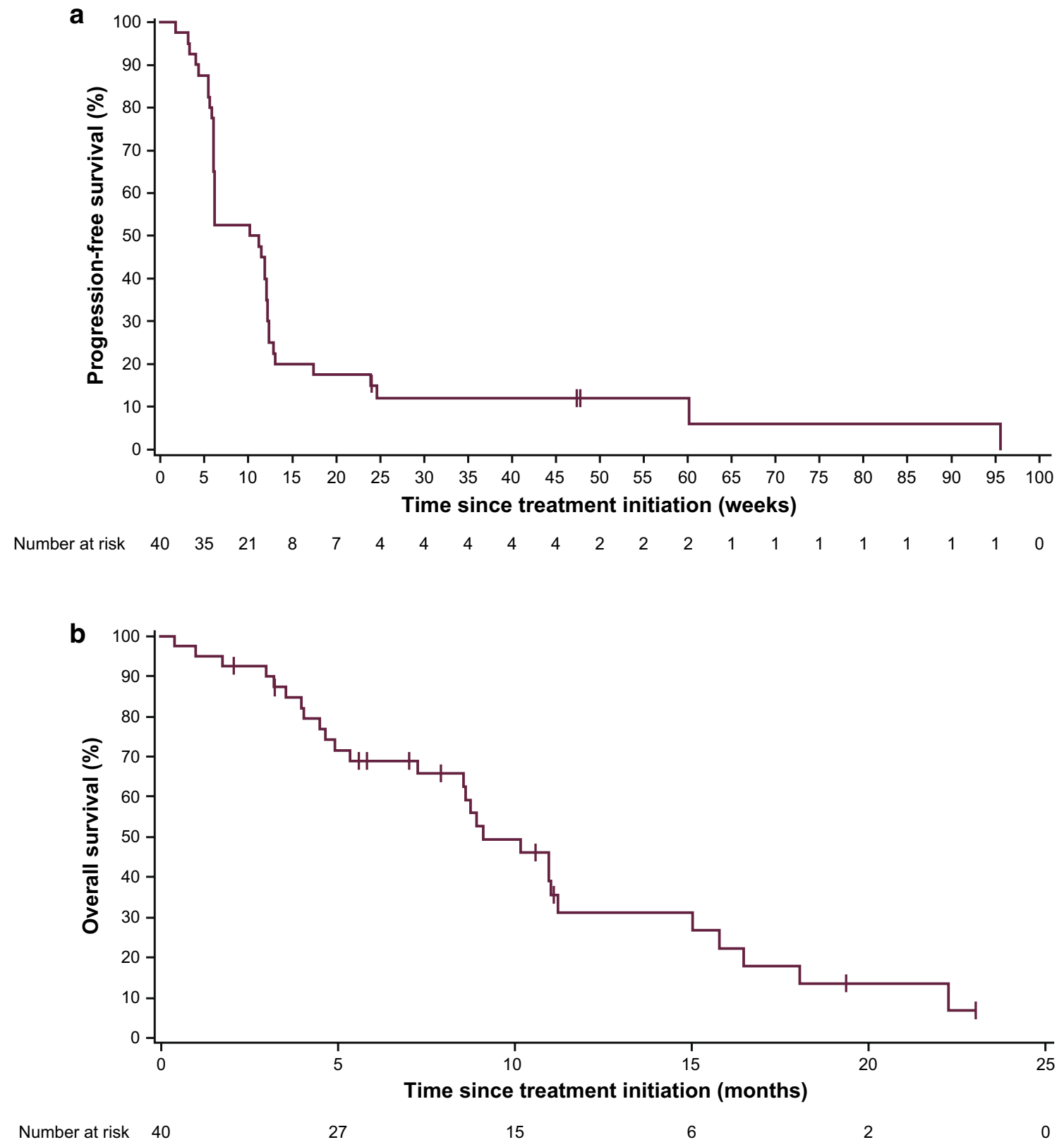

Fig. 2 Kaplan-Meier estimates of a progression-free survival and b overall survival in patients with GC/GEJC in the dose-expansion cohort $(n=40)$ 
from those used in trials of other anti-PD-1 or PD-L1 agents. Moreover, PD-L1 status in this study was determined based on tumor cell expression only, whereas in the KEYNOTE-059 study of pembrolizumab, PD-L1 status was based on expression on tumor cells and immune cells (ie, combined proportion score) [21], hampering any cross-trial comparison of efficacy trends in GC/GEJC based on PD-L1 status.

As discussed earlier, 2 global phase 3 trials in patients with previously treated advanced GC/GEJC (KEYNOTE-061 and JAVELIN Gastric 300) that compared anti-PD-1/PD-L1 antibody treatment (pembrolizumab or avelumab) with standard second-line or third-line chemotherapy did not meet their primary endpoints $[22,30]$. However, several phase 3 studies are ongoing to investigate alternative uses of checkpoint inhibitors in the treatment of GC/GEJC. For example, an ongoing phase 3 trial (JAVELIN Gastric 100) is assessing switchmaintenance treatment with avelumab vs continuation of first-line chemotherapy. Other ongoing phase 3 studies in patients with advanced GC/GEJC include a trial of secondline pembrolizumab monotherapy vs paclitaxel in Asian patients (KEYNOTE-063), a trial of pembrolizumab as first-line treatment in combination with chemotherapy (KEYNOTE-062), a trial of nivolumab in combination with chemotherapy as first-line treatment in Asian patients (ATTRACTION-04), and a trial of first-line nivolumab plus ipilimumab or chemotherapy vs chemotherapy alone (CheckMate-649). Results from ongoing studies will help to define an appropriate role for checkpoint inhibitors in the treatment of GC/GEJC.

Acknowledgements We thank the patients and their families, the investigators, the coinvestigators, and the study teams at each of the participating centers and at Merck KGaA, Darmstadt, Germany and Merck Serono, Tokyo, Japan (a Japanese subsidiary of Merck KGaA, Darmstadt, Germany). We also thank Vikram Chand (formerly of EMD Serono) for his medical guidance during the conduct of the trial. This trial was sponsored by Merck $\mathrm{KGaA}$ and is part of an alliance between Merck KGaA and Pfizer (New York, NY, USA). Medical writing support was provided by ClinicalThinking and funded by Merck KGaA and Pfizer.

\section{Compliance with ethical standards}

Conflict of interest TD has received research funding from AbbVie, Astellas Pharma, Bristol-Myers Squibb, Boehringer Ingelheim, Celgene, Chugai Pharma, Daiichi Sankyo, IQVIA, Janssen, Kyowa Hakko Kirin, Lilly Japan, MSD, Merck Serono, Novartis, Pfizer, Sumitomo Group Takeda, and Taiho Pharmaceutical, and reports consulting roles for Amgen, Chugai Pharma, Daiichi Sankyo, Kyowa Hakko Kirin, Lilly Japan, MSD, Sumitomo Dainippon, and Taiho Pharmaceutical. SI reports honoraria from Chugai Pharma and Takeda, and has received research funding from AbbVie, AstraZeneca, Astellas, Bayer, Chugai Pharma, Daiichi Sankyo, Eisai, Lilly, Merck Serono, Nano Carrier, Novartis, Ono Pharmaceutical, Otsuka, Sanofi, Sumitomo Dainippon, Taiho Pharmaceutical, and Teijin. KM reports honoraria from Chugai
Pharma, Merck Serono, Takeda, Taiho Pharmaceutical, and Yakult. TS reports honoraria and consulting or advisory roles for Bristol-Myers Squibb, Chugai Pharma, and Merck Serono; TS also reports consulting or advisory roles for Lilly and has received research funding from Chugai Pharma and Yakult. SH reports honoraria from Novartis, Taiho Pharmaceutical, Takeda, and Yakult, and reports consulting or advisory roles for Lilly and Yakult. TE has received research funding from Merck Serono. TN reports honoraria from Merck Serono. HH reports honoraria from Chugai Pharma, Lilly, Merck Serono, Ono Pharmaceutical, Taiho Pharmaceutical, Takeda, and Yakult, and reports consulting or advisory roles from Chugai Pharma, MSD, Merck Serono, and Ono Pharmaceutical. $\mathrm{HH}$ has received research funding from AstraZeneca, Boehringer Ingelheim, Chugai Pharma, Daiichi Sankyo, Eisai, Incyte, Lilly, LSK BioPharma, MSD, Merck Serono, Ono Pharmaceutical, Pfizer, Sumitomo Dainippon, Taiho Pharmaceutical, and Takeda. NM and SO have no disclosures to report. YK reports honoraria and speaker services for Bayer, Novartis, and Pfizer; YK also reports speaker services for Chugai Pharma, Lilly, Merck Serono, and Taiho Pharmaceutical, and research funding from Bayer, Chugai Pharma, Lilly, MSD, Novartis, Ono Pharmaceutical, Taiho, and Yakult. YS reports honoraria from Bayer Yakuhin, Chugai Pharma, Daiichi Sankyo, Lilly, Merck Serono, Novartis, Ono Pharmaceutical, Taiho Pharmaceutical, Takeda, and Yakult; YS has also received research funding from Chugai Pharma, Lilly, Merck Serono, MSD, and Taiho Pharmaceutical. SS and MW are employees of Merck Serono.

Ethical standards The trial was conducted in accordance with the ethics principles of the Declaration of Helsinki and the International Council on Harmonisation Guidelines on Good Clinical Practice (GCP) or Japanese Ministerial Ordinance on GCP. The protocol was approved by the institutional review board or independent ethics committee of each center. All patients provided written informed consent before enrollment.

Open Access This article is distributed under the terms of the Creative Commons Attribution 4.0 International License (http://creativeco mmons.org/licenses/by/4.0/), which permits unrestricted use, distribution, and reproduction in any medium, provided you give appropriate credit to the original author(s) and the source, provide a link to the Creative Commons license, and indicate if changes were made.

\section{References}

1. Ferlay J, Soerjomataram I, Ervik M, Dikshit R, Eser S, Mathers C, et al. GLOBOCAN 2012 v1.0, Cancer incidence and mortality worldwide: IARC CancerBase No. 11 [Internet]. Lyon, France: International Agency for Research on Cancer; 2013. http://globo can.iarc.fr/. Accessed 17 Aug 2018.

2. Ferlay J, Soerjomataram I, Dikshit R, Eser S, Mathers C, Rebelo $\mathrm{M}$, et al. Cancer incidence and mortality worldwide: sources, methods and major patterns in GLOBOCAN 2012. Int J Cancer. 2015;136:E359-86.

3. Wang J, Sun Y, Bertagnolli MM. Comparison of gastric cancer survival between Caucasian and Asian patients treated in the United States: results from the Surveillance Epidemiology and End Results (SEER) database. Ann Surg Oncol. 2015;22:2965-71.

4. Chen Y, Haveman JW, Apostolou C, Chang DK, Merrett ND. Asian gastric cancer patients show superior survival: the experiences of a single Australian center. Gastric Cancer. 2015;18:256-61.

5. Theuer CP, Kurosaki T, Ziogas A, Butler J, Anton-Culver H. Asian patients with gastric carcinoma in the United States exhibit 
unique clinical features and superior overall and cancer specific survival rates. Cancer. 2000;89:1883-92.

6. Kim J, Sun CL, Mailey B, Prendergast C, Artinyan A, Bhatia S, et al. Race and ethnicity correlate with survival in patients with gastric adenocarcinoma. Ann Oncol. 2010;21:152-60.

7. Buas M, Vaughan TL. Epidemiology and risk factors for gastroesophageal junction tumors: understanding the rising incidence of this disease. Semin Radiat Oncol. 2013;23:3-9.

8. National Comprehensive Cancer Network. NCCN Clinical Practice Guidelines in Oncology: Esophageal and Esophagogastric Junction Cancers. V2.2018. https://www.nccn.org/professionals/ physician_gls/pdf/esophageal.pdf. Accessed 1 Sept 2018.

9. National Comprehensive Cancer Network. NCCN Clinical Practice Guidelines in Oncology: Gastric Cancer. V2.2018. https ://www.nccn.org/professionals/physician_gls/pdf/gastric.pdf. Accessed 1 Sept 2018.

10. Smyth EC, Verheij M, Allum W, Cunningham D, Cervantes A, Arnold D; ESMO Guidelines Committee. Gastric cancer: ESMO Clinical Practice Guidelines for diagnosis, treatment and followup. Ann Oncol. 2016;27:v38-49.

11. Japanese Gastric Cancer Association. Japanese gastric cancer treatment guidelines 2014 (ver.4). Gastric Cancer. 2017;20:1-19.

12. Wilke H, Muro K, Van Cutsem E, Oh SC, Bodoky G, Shimada Y, et al. Ramucirumab plus paclitaxel versus placebo plus paclitaxel in patients with previously treated advanced gastric or gastrooesophageal junction adenocarcinoma (RAINBOW): a doubleblind, randomised phase 3 trial. Lancet Oncol. 2014;15:1224-35.

13. Kothari N, Almhanna K. Current status of novel agents in advanced gastroesophageal adenocarcinoma. J Gastrointest Oncol. 2015;6:60-74.

14. Boger C, Behrens HM, Mathiak M, Kruger S, Kalthoff H, Rocken C. PD-L1 is an independent prognostic predictor in gastric cancer of Western patients. Oncotarget. 2016;7(17):24269-83.

15. Kim JW, Nam KH, Ahn SH, Park DJ, Kim HH, Kim SH, et al. Prognostic implications of immunosuppressive protein expression in tumors as well as immune cell infiltration within the tumor microenvironment in gastric cancer. Gastric Cancer. 2016;19:42-52.

16. Qing Y, Li Q, Ren T, Xia W, Peng Y, Liu GL, et al. Upregulation of PD-L1 and APE1 is associated with tumorigenesis and poor prognosis of gastric cancer. Drug Des Devel Ther. 2015;9:901-9.

17. Dai C, Wang M, Lu J, Dai Z, Lin S, Yang P, et al. Prognostic and predictive values of PD-L1 expression in patients with digestive system cancer: a meta-analysis. Onco Targets Ther. 2017;10:3625-34.

18. Wu P, Wu D, Li L, Chai Y, Huang J. PD-L1 and survival in solid tumors: a meta-analysis. PLoS One. 2015;10:e0131403.

19. Takaya S, Saito H, Ikeguchi M. Upregulation of immune checkpoint molecules, PD- 1 and LAG-3, on CD4+ and CD8+ T cells after gastric cancer surgery. Yonago Acta Med. 2015;58:39-44.

20. Kang YK, Boku N, Satoh T, Ryu MH, Chao Y, Kato K, et al. Nivolumab in patients with advanced gastric or gastro-oesophageal junction cancer refractory to, or intolerant of, at least two previous chemotherapy regimens (ONO-4538-12, ATTRAC TION-2): a randomised, double-blind, placebo-controlled, phase 3 trial. Lancet. 2017;390:2461-71.

21. Fuchs CS, Doi T, Jang RW, Muro K, Satoh T, Machado M, et al. Safety and efficacy of pembrolizumab monotherapy in patients with previously treated advanced gastric and gastroesophageal junction cancer: phase 2 clinical KEYNOTE-059 trial. JAMA Oncol. 2018;4:e180013.
22. Shitara K, Ozguroglu M, Bang YJ, Bartolomeo MD, Mandala M, Ryu MH, et al. Pembrolizumab versus paclitaxel for previously treated, advanced gastric or gastro-oesophageal junction cancer (KEYNOTE-061): a randomised, open-label, controlled, phase 3 trial. Lancet. 2018;392:123-33.

23. Heery CR, O'Sullivan-Coyne G, Madan RA, Cordes LM, Rajan A, Rauckhorst M, et al. Avelumab for metastatic or locally advanced previously treated solid tumours (JAVELIN Solid Tumor): a phase 1a, multicohort, dose-escalation trial. Lancet Oncol. 2017;18:587-97.

24. Vandeveer AJ, Fallon JK, Tighe R, Sabzevari H, Schlom J, Greiner JW. Systemic immunotherapy of non-muscle invasive mouse bladder cancer with avelumab, an anti-PD-L1 immune checkpoint inhibitor. Cancer Immunol Res. 2016;4:452-62.

25. Boyerinas B, Jochems C, Fantini M, Heery CR, Gulley JL, Tsang $\mathrm{KY}$, et al. Antibody-dependent cellular cytotoxicity activity of a novel anti-PD-L1 antibody avelumab (MSB0010718C) on human tumor cells. Cancer Immunol Res. 2015;3:1148-57.

26. Bavencio (avelumab) injection [package insert]. Tokyo, Japan: Merck Serono Co.; 2017.

27. Bavencio (avelumab) prescribing information. Rockland, MA, USA: EMD Serono, Inc; 2018.

28. Bavencio (avelumab) [summary of product characteristics]. Darmstadt, Germany: Merck KGaA; 2018.

29. Chung H, Arkenau HT, Lee J, Rha S, Oh D, Wyrwicz L, et al. Avelumab (anti-PD-L1) as first-line maintenance (1L mn) or second-line (2L) therapy in patients with advanced gastric or gastroesophageal junction cancer (GC/GEJC): updated phase Ib results from the JAVELIN Solid Tumor trial. Cancer Res. 2018;78(13 suppl):CT111 (abstract).

30. Bang YJ, Ruiz EY, Van Cutsem E, Lee KW, Wyrwicz L, Schenker M, et al. Phase III, randomised trial of avelumab versus physician's choice of chemotherapy as third-line treatment of patients with advanced gastric or gastro-oesophageal junction cancer: primary analysis of JAVELIN Gastric 300. Ann Oncol. 2018;29:2052-60.

31. Feng Z, Schlichting M, Helwig C, Chand VK, Gelb A, Jin H, et al. Comparative study of two PD-L1 expression assays in patients with non-small cell lung cancer (NSCLC). J Clin Oncol. 2017;35(15 suppl):e20581 (abstract).

32. Gulley JL, Rajan A, Spigel DR, Iannotti N, Chandler J, Wong DJL, et al. Avelumab for patients with previously treated metastatic or recurrent non-small-cell lung cancer (JAVELIN Solid Tumor): dose-expansion cohort of a multicentre, open-label, phase $1 \mathrm{~b}$ trial. Lancet Oncol. 2017;18:599-610.

33. Wolchok JD, Hoos A, O'Day S, Weber JS, Hamid O, Lebbe C, et al. Guidelines for the evaluation of immune therapy activity in solid tumors: immune-related response criteria. Clin Cancer Res. 2009;15:7412-20.

34. Feldschuh J, Enson Y. Prediction of the normal blood volume. Relation of blood volume to body habitus. Circulation. 1977;56:605-12.

35. Muro K, Chung HC, Shankaran V, Geva R, Catenacci D, Gupta S, et al. Pembrolizumab for patients with PD-L1-positive advanced gastric cancer (KEYNOTE-012): a multicentre, open-label, phase 1b trial. Lancet Oncol. 2016;17:717-26.

36. Janjigian YY, Bendell J, Calvo E, Kim JW, Ascierto PA, Sharma $\mathrm{P}$, et al. CheckMate-032 study: efficacy and safety of nivolumab and nivolumab plus ipilimumab in patients with metastatic esophagogastric cancer. J Clin Oncol. 2018;36:2836-44. 